\title{
Chronic Bilateral Cochlear Implant Stimulation Partially Restores Neural Binaural Sensitivity in Neonatally-Deaf Rabbits
}

\author{
${ }^{(-)}$Woongsang Sunwoo, ${ }^{1,2,3}{ }^{\circledR}$ Bertrand Delgutte, ${ }^{1,2}$ and ${ }^{\circledR}$ Yoojin Chung ${ }^{1,2}$ \\ ${ }^{1}$ Eaton-Peabody Laboratories, Massachusetts Eye and Ear, Boston, Massachusetts 02114, ${ }^{2}$ Department of Otolaryngology-Head and Neck Surgery, \\ Harvard Medical School, Boston, Massachusetts 02115 , and ${ }^{3}$ Department of Otolaryngology, Gil Medical Center, Gachon University College of \\ Medicine, Incheon 21565, South Korea
}

Cochlear implant (CI) users with a prelingual onset of hearing loss show poor sensitivity to interaural time differences (ITDs), an important cue for sound localization and speech reception in noise. Similarly, neural ITD sensitivity in the inferior colliculus (IC) of neonatally-deafened animals is degraded compared with animals deafened as adults. Here, we show that chronic bilateral CI stimulation during development can partly reverse the effect of early-onset deafness on ITD sensitivity. The prevalence of ITD sensitive neurons was restored to the level of adult-deaf (AD) rabbits in the early-deaf rabbits of both sexes that received chronic stimulation and behavioral training with wearable bilateral sound processors during development. We also found a partial improvement in neural ITD sensitivity in the early-deaf and stimulated rabbits compared with unstimulated rabbits. In contrast, chronic CI stimulation did not improve temporal coding in early-deaf rabbits. The present study is the first report showing functional restoration of ITD sensitivity with CI stimulation in single neurons and highlights the importance of auditory experience during development on the maturation of binaural circuitry.

Key words: binaural hearing; cochlear implant; deafness; development; interaural time difference; plasticity

Significance Statement

Although cochlear implants (CI) are highly successful in providing speech reception in quiet for many profoundly deaf people, $\mathrm{CI}$ users still face difficulty in noisy everyday environment. This is partly because of their poor sensitivity to differences in the timing of sounds arriving at the two ears [interaural time differences (ITDs)], which help to identify where the sound is coming from. This problem is especially acute in those who lost hearing early in life. Here, we present the first report that sensitivity of auditory neurons to ITDs is restored by CI stimulation during development in an animal model of neonatal deafness. These findings highlight the importance of providing early binaural auditory experience with CIs in deaf children.

\section{Introduction}

Although cochlear implantation provides effective treatment for profound deafness, both adult and pediatric cochlear implant (CI) users still face challenges understanding conversations in everyday noisy acoustic environments. Normal-hearing $(\mathrm{NH})$ listeners benefit from using binaural cues for sound localization

\footnotetext{
Received May 4, 2020; revised Feb. 12, 2021; accepted Feb. 21, 2021.

Author contributions: B.D. and Y.C. designed research; W.S. and Y.C. performed research; W.S. and Y.C. analyzed data; Y.C. wrote the paper.

This work was supported by the National Institutes of Health Grant R01 DC005775. We thank Ken Hancock, Ishmael Stefanov-Wagner, Alice Gelman, Joseph Wagner, Stephanie Ventura, and Marie Ortega for technical assistance; Hugh Curtin and Renee Mitchell for assistance in radiographic imaging; Kevin Franck for advice on programming the cochlear implant sound processors; and Zachary Smith at Cochlear Ltd for providing the cochlear implant sound processors and software.

The authors declare no competing financial interests.

Correspondence should be addressed to Yoojin Chung at yoojin.chung@gmail.com.

https://doi.org/10.1523/JNEUROSCI.1076-20.2021

Copyright $\odot 2021$ the authors
}

and understanding speech in noise. A growing number of CI users, including children, receive implants in both ears so they can benefit from these binaural cues as well. However, binaural performance of bilateral CI users is poorer than that of $\mathrm{NH}$ listeners, particularly for tasks that require sensitivity to interaural time differences (ITDs; van Hoesel and Tyler, 2003; van Hoesel et al., 2009; Salloum et al., 2010; Laback et al., 2015). As a result, bilateral CI listeners' abilities to localize sound sources and understand speech in everyday acoustic environments are worse than those of NH listeners' (Murphy and O'Donoghue, 2007; Litovsky et al., 2009). In addition, CI users' sensitivity to ITD is highly dependent on stimulus parameters such as pulse rate and intensity (Kan and Litovsky, 2015; Laback et al., 2015). This problem is especially acute in patients who experience earlyonset hearing loss. CI users with prelingual onset of hearing loss who received CIs as adults either cannot perceive ITDs or perform very poorly in ITD discrimination tasks, whereas they are sensitive to interaural level differences (ILDs; Litovsky et al., 
2010). Single neurons in the central auditory pathway show similar limitations in how they encode bilateral CI stimulation. Degraded neural ITD sensitivity with bilateral CI stimulation has been observed in the auditory midbrain (Hancock et al., 2010) and auditory cortex (Tillein et al., 2010) of congenitally deaf cats under anesthesia, and auditory midbrain of neonatally-deafened rabbits in an unanesthetized preparation (Chung et al., 2019).

Whether chronic auditory stimulation through bilateral CIs can restore ITD sensitivity in central auditory neurons is unknown. Children who received bilateral CIs at an early age show some improvement in perception of binaural cues, although their ITD sensitivity remains poorer than that of $\mathrm{NH}$ children (Gordon et al., 2014). Similarly, evoked potential studies in congenitally deaf children who received simultaneous bilateral CIs show that auditory experience with current implant devices does not fully reverse the effects of early deafness (Easwar et al., 2017). Studies in congenitally deaf cats have shown that chronic bilateral CI stimulation during development can restore a more normal distribution of inhibitory inputs to neurons in the medial superior olive (MSO), one of the brainstem nuclei where ITD processing first takes place (Tirko and Ryugo, 2012). To what extent neural ITD sensitivity can be restored with chronic bilateral CI stimulation is unknown.

We developed a novel preparation for neonatal deafening, early cochlear implantation, and chronic stimulation with wearable bilateral processors in rabbits. With this preparation, we investigated whether chronic bilateral CI stimulation during development can reverse the effect of early-onset deafness on neural ITD sensitivity and temporal coding in the auditory midbrain. Neurophysiological recordings were made from unanesthetized animals because neural temporal coding and ITD sensitivity of auditory midbrain neurons better match perceptual results from human CI subjects in unanesthetized animals than in anesthetized animals (Chung et al., 2014, 2016). We found that chronic bilateral CI stimulation during development can partly reverse the degradation in neural ITD sensitivity resulting from early-onset deafness. The effect was most pronounced in response to high-rate stimulation. However, there was no reversal in the effect of early-onset deafness on neural temporal coding and survival of spiral ganglion neurons (SGN).

\section{Materials and Methods}

\section{Animals and experiment design}

All experiments were approved by the animal care and use committee of Massachusetts Eye and Ear. Four rabbits (three females, and one male) were deafened as neonates and implanted at two months of age (Fig. 1). Chronic stimulation with wearable bilateral processors commenced one to two weeks after implantation. Neurophysiological data from the inferior colliculus (IC) were collected from all four implanted rabbits starting at six months of age and for durations of three to seven months.

Neural data from these four early-deafened and chronically stimulated (ED-CS) rabbits were compared with reanalyzed data from four adult-deafened (AD) rabbits (Chung et al., 2016) and three early-deafened and unstimulated (ED-US) rabbits (Chung et al., 2019) used in previous studies. The $\mathrm{AD}$ rabbits were bilaterally deafened and implanted at $8-10$ months of age and studied neurophysiologically for approximately six months. These rabbits were deafened during the implantation surgery by injection of distilled water into scala tympani to cause hair cell death through osmotic stress (Chung et al., 2014, 2019). The three ED-US rabbits were deafened by daily injections of neomycin and implanted at two to three months of age as the ED-CS rabbits. Here, we only describe the methods in detail for the ED-CS group because the methods for other groups were reported previously. A detailed history of all surgical and experimental procedures in all rabbits is given in Table 1.

\section{Neonatal deafening}

Newborn rabbits were deafened by daily subcutaneous injections of neomycin sulfate $(60 \mathrm{mg} / \mathrm{kg} / \mathrm{d})$ from the first postnatal day (Chung et al., 2019), before the onset of hearing which occurs at P7 in this species (Foss and Flottorp, 1974). Auditory brainstem responses (ABRs) to acoustic clicks were measured at postnatal day $>$ P22 under sedation ( $2 \mathrm{mg} / \mathrm{kg}$ midazolam, i.m., $10 \mathrm{mcg} / \mathrm{kg}$ fentanyl, i.m.). In all neonatallydeafened rabbits, the injections were discontinued after verifying that no measurable ABRs could be obtained at $110 \mathrm{~dB}$ SPL.

\section{Surgical procedures}

All surgical procedures were performed under anesthesia $(6 \mathrm{mg} / \mathrm{kg}$ xylazine, s.c., $35 \mathrm{mg} / \mathrm{kg}$ ketamine, i.m.; then maintained by isoflurane $2.5 \%$ delivered via facemask in $0.8 \mathrm{l} / \mathrm{min} \mathrm{O}_{2}$ ) and strict aseptic conditions.

At two months of age, the rabbits were implanted bilaterally with intracochlear electrode arrays. The method of cochlear implantation was the same as described in detail previously (Chung et al., 2014, 2016, 2019). The bulla was exposed via a retroauricular approach, and opened with a bone drill to visualize the basal turn of the cochlea and round window. The round window was drilled and enlarged enough to achieve full insertion of an HL-8 eight contact electrode array (Cochlear Ltd.) without resistance. The return electrode was placed in the auricular muscle. The opening of the enlarged round window and the bulla were sealed with a small piece of muscle and bone cement, respectively. CI connectors were attached to the skull with stainless steel screws and dental cement. Immediately after the CI surgery, postoperative imaging, including skull $\mathrm{x}$-ray and computed tomography, was performed to detect possible electrode misplacements and to assess insertion depth. Electrode misplacement such as fold-over, loop, kinking, and scalar crossing was not observed in any case.

When the animals reached six months of age, which corresponds to sexual maturity in rabbits (Foote and Carney, 2000), a brass bar was attached to the skull and a cylindrical barrier $(1.5-2 \mathrm{~cm}$ in diameter) for a sterile portal to a subsequent craniotomy was built with dental cement. After recovery from headpost surgery (approximately one week), rabbits were habituated to the experimental setup until they could sit quietly for $2-3 \mathrm{~h}$ with the head fixed while receiving electrical stimulation. After the habituation period (one to two weeks), a third surgery was performed to allow insertion of a recording electrode into the IC. Two small craniotomies were made, one on each parietal bone inside the cylindrical barrier. The exposed dura of the occipital cortex was covered with a topical 
Table 1. Summary of deafness, recording history, and histologic processing for each animal

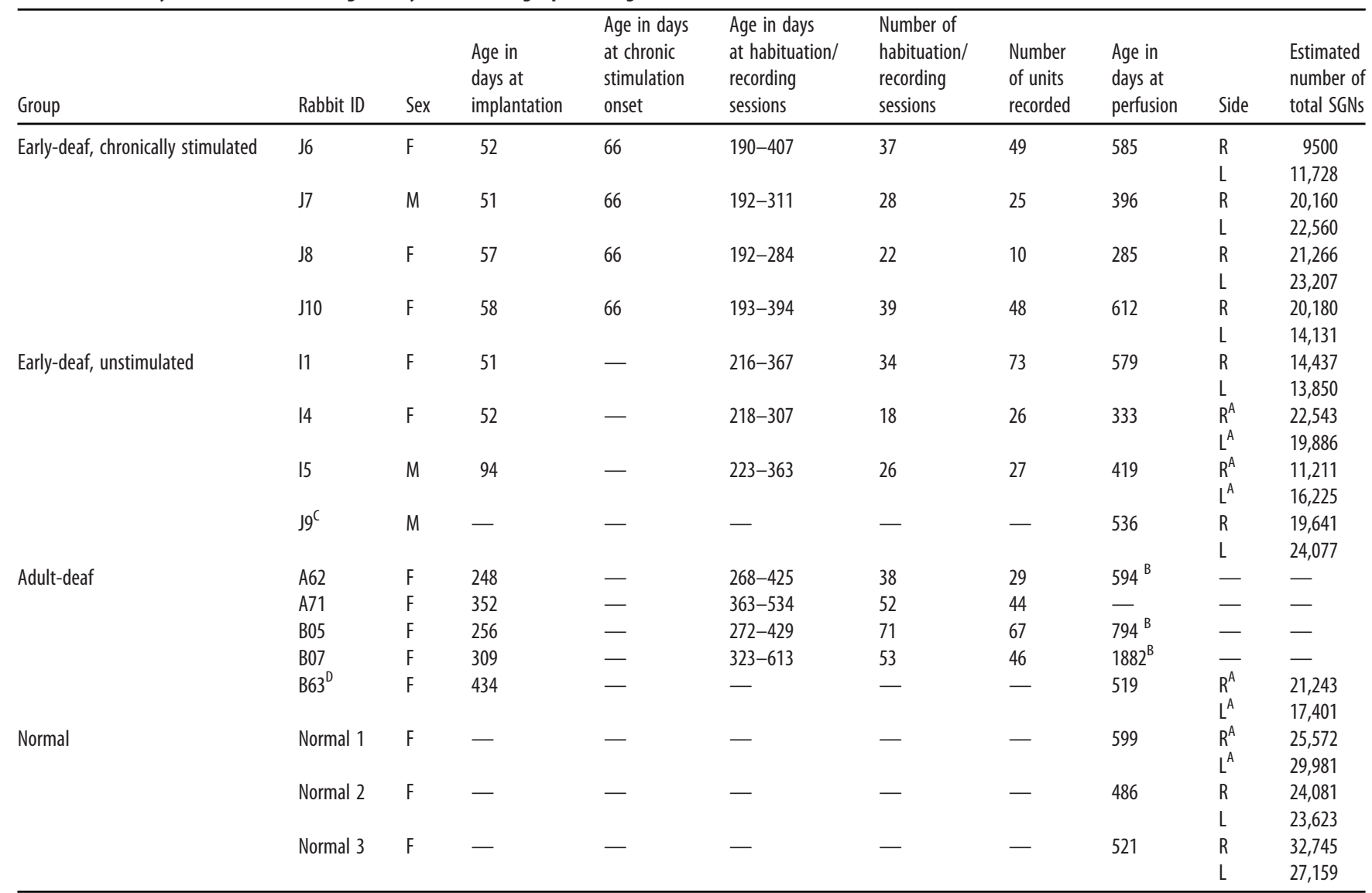

For adult deafened rabbits, the onset of deafness is the same as the age at implantation (i.e., all AD rabbits were deafened during the (I surgery). A, Embedded in celloidin and stained with hematoxylin and eosin. All other cochleas were embedded in araldite and stained with toluidine blue. B, Only the brain tissue was processed. C, This animal was neonatally deafened but not implanted. D, No electrophysiological data included in the current study was collected from this rabbit.

antibiotic and the cylinder was sealed with dental impression material. Neurophysiological recordings through the craniotomy commenced after $3 \mathrm{~d}$ of recovery.

\section{Chronic stimulation with bilateral wearable processors}

One week after the implant surgery, the CIs were activated. The implant processors (Nucleus CP800, Cochlear Ltd.) were programmed using the Custom-Sound programming software (Cochlear Ltd.) while the rabbits were awake. At the first step in programming, the impedance and the electrically evoked compound action potential (ECAP) were measured for each intracochlear electrode contact to assess the effectiveness of cochlear implantation. For the next step in programming, the numbers of activated channels and electrode pairs were matched between the two ears based on radiographic results. Figure 2, bottom panel, shows an $\mathrm{x}$ ray image of one of the two animals (J7 and J8), for which a symmetric insertion was achieved. In the other two animals, there was an interaural mismatch as shown in the example in Figure 2, top panel. In both animals, the interaural mismatch was about two electrode intervals with the right implant inserted deeper in J6 (Fig. 2A) and left in J10. When full insertion is achieved, the 7-mm-long electrode array reaches the characteristic frequency region of $\sim 2 \mathrm{kHz}$ (Ramprashad et al., 1984). The ECAP threshold profile and animal's behavioral response were used to estimate the maximum stimulation levels and expected threshold levels for each electrode assuming a typical electrical dynamic range $(6.3 \mathrm{~dB}$ or 40 current levels in nucleus devices).

Electrode impedances and ECAP thresholds, as indicators of implant and physiological function, were measured weekly and monthly, respectively. Electrodes with abnormally high impedance and shorted electrodes were deactivated and the sound processors were reprogrammed to achieve symmetry between the two ears. At the time of activation, the number of active bilateral electrode pairs ranged from 6 to 7 across the four animals. Impedances and ECAP thresholds were stable for seven months from implantation for all animals. After 30 weeks, two and four pairs of electrodes were deactivated in two animals (J6 and J7, respectively). At the 39th week, one pair of electrodes was deactivated in J10 and an additional pair in J7. No electrode was deactivated in J8. No shift in the electrode array placement was observed in follow-up x-ray images taken four months postimplantation.

We used an experimental fundamental asynchronous stimulus timing (FAST) strategy specifically designed to deliver ITD cues (Smith, 2010; Smith et al., 2014; Hossain and Goldsworthy, 2018) to chronically stimulate the cochleas. The FAST strategy uses current pulses that are precisely timed to local maxima of the sound envelope in each frequency channel, resulting in implicit synchronization between the carrier pulses at the two ears and much lower average pulse rates than in the continuous interleaved sampling (CIS) strategy used in most clinical devices.

Chronic stimulation commenced approximately one to two weeks after CI surgery. Passive sound stimulation took place in the rabbits' home cage in the animal care facility. Stimulation included environmental sounds associated with normal activities in the animal care facility and sounds of nature (Cornell Lab of Ornithology, 2001; Elliott and Hershberger, 2007) played via a loudspeaker. The overall sound level in the home cage ranged 60-70 dB SPL. During the stimulation sessions, rabbits wore a modified CI device pair (Nucleus CP800 sound processor and CI emulator T51107, Cochlear Ltd.) in a backpack with the outside auxiliary microphones positioned posterior to the ipsilateral ear generating binaural cues similar to the biological range. Environmental stimulation was given for up to $5 \mathrm{~h} / \mathrm{d}, 5 \mathrm{~d} /$ week.

\section{Behavioral task}

In addition to the passive environmental stimulation, active behavioral training via operant conditioning in a virtual sound localization task was 
A
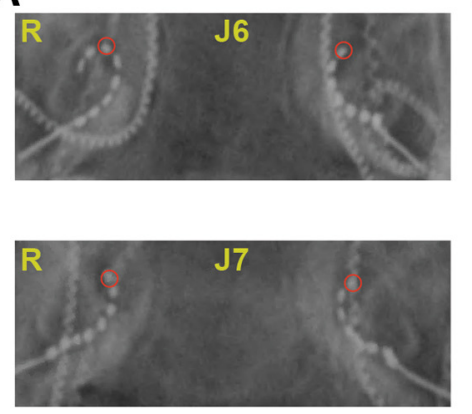

B

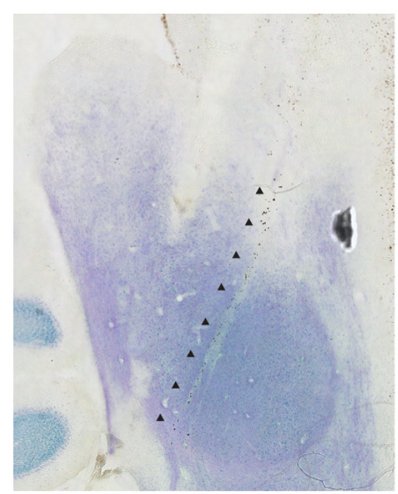

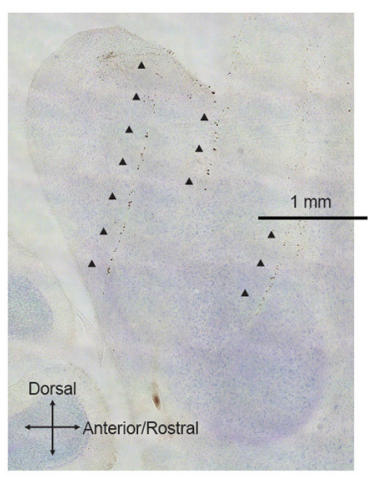

Figure 2. A, X-ray images of the cochleas in two ED-CS rabbits, one with asymmetric insertion of the $\mathrm{Cl}$ array (top) and one with symmetric insertion (bottom). $\boldsymbol{B}$, Electrode tracks (triangle marks) in two sagittal sections of the IC of one ED-CS rabbit (J6).

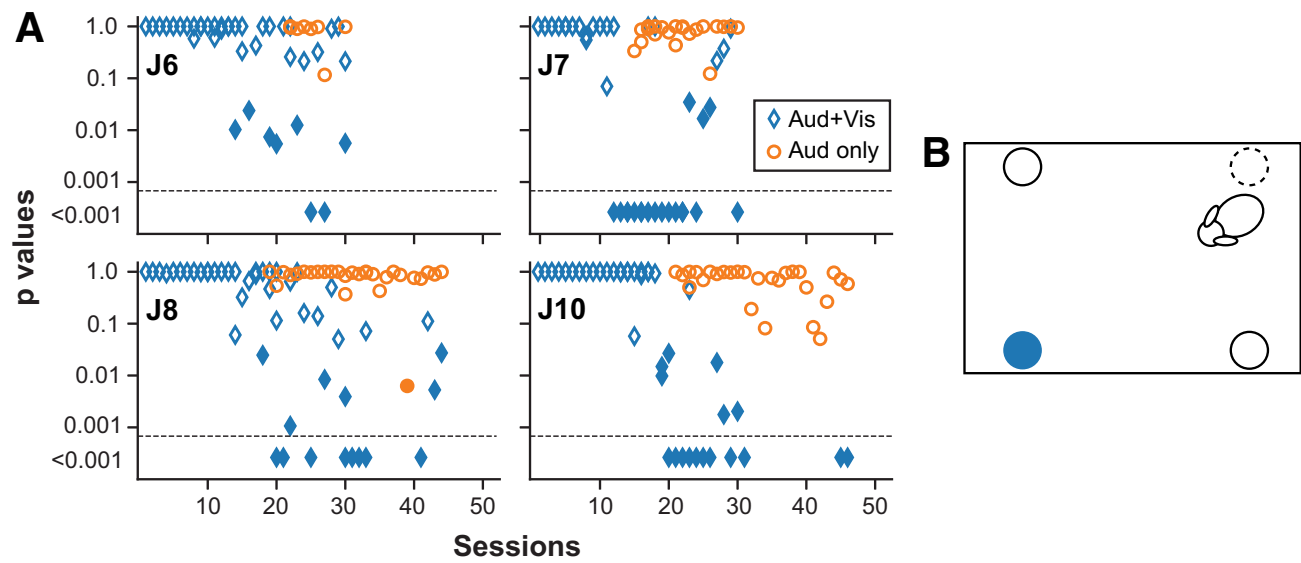

Figure 3. A, Performance of ED-CS rabbits in the behavioral task quantified as $p$ values for binomial tests comparing the percentage of correctly identified targets to chance. Filled marks represent sessions with success rate statistical significance $(p=0.05)$. $\boldsymbol{B}$, Scheme of the setting. A target (filled) is selected randomly excluding the one nearest to the animal (dashed edge) on each trial.

performed $3 \mathrm{~d} /$ week, for up to $3 \mathrm{~h} / \mathrm{d}$, in a sound-treated booth where the rabbit was able to move freely. Animals were food restricted after recovering from the CI surgery to maintain a target weight of $80 \%$ of the weight estimated from the standard growth rate of Dutch-belted rabbits. The task was to find a virtual sound target associated with one of two to four food bowls connected to pellet feeders. Two to four targets were placed at each corner of a rectangular arena (Fig. $3 B$ ). The sound target location was randomly selected (excluding the one nearest to the animal) on each trial by a computer. A stimulus was presented continuously throughout the duration of each trial. A food pellet was given as a reward if the rabbit successfully reached the correct target within $60 \mathrm{~s}$. A trial was considered failed if the rabbit either reached an incorrect target or failed to reach any target after $1 \mathrm{~min}$. At the end of a failed trial, the stimulus was discontinued and light in the booth was turned off for $10 \mathrm{~s}$ before a new trial could start.

During the behavioral task, the rabbits wore the CI processors connected to a Bluetooth audio receiver placed within the sound processor backpack. A Bluetooth transmitter emitted a continuous binaural amplitude-modulated pure tone that was transformed by the processor into a pulse train at the $100-\mathrm{Hz}$ modulation rate delivered to a pair of monopolar electrodes with appropriate binaural cues. Specifically, the computer monitored the rabbit's position and orientation within the room using a video camera, and continuously updated the ITD $(-750$ to $+750 \mu \mathrm{s})$, the ILDs $(-5$ to $+5 \mathrm{~dB})$ and the mean level so the sound would appear to originate from the target. We used a wider range of ITDs than the physiologically relevant range for rabbits because humans CI users are able to lateralize ITDs as large as twice their physiological limit (Baumgärtel et al., 2017).
Initially, the rabbits were trained to find the target using both auditory cues and visual cues produced by a light emitting diode placed near the target location. When the rabbits performed the task with abovechance accuracy using audiovisual stimulation, they were weaned off the visual cues and trained with auditory stimuli alone. Because none of the rabbits showed any significant progress in performance with auditory stimuli alone after 30-45 sessions, the behavioral training sessions were discontinued after three months.

\section{Electrophysiological methods}

Single-unit recordings

All neurophysiological recording procedures were the same in the three animal groups. Polyimide insulated platinum/iridium linear microelectrode arrays with four to six contacts $(150-\mu \mathrm{m}$ spacing between contacts, $12.5-\mu \mathrm{m}$ site diameter; MicroProbes) were used to record single-unit activity from the IC. Recording electrodes were advanced in a dorsoventral direction through the occipital cortex down to the IC, which was identified by entrained multiunit activity to the search stimuli. We sampled neurons across all depths where this background entrainment was observed. The point of entry of the electrode within the craniotomy was intentionally varied to widely sample the IC.

To reduce the electrical stimulus artifact, recordings were made differentially between one electrode contact showing clear spike activity and a local reference obtained by averaging the signals from the remaining contacts. Signals from the recording electrodes were acquired by a unity gain headstage (HST/16050; Plexon) and then amplified (PBX2; Plexon) and lightly filtered $(100-8000 \mathrm{~Hz})$ to avoid prolonging the stimulus artifact. The conditioned signals were sampled at $100 \mathrm{kHz}$ using a 12-bit A/D converter. For low pulse rates $(<200 \mathrm{pps})$, the stimulus 
artifact was removed by a gate-and- interpolate technique (Heffer and Fallon, 2008) that can result in some spike detection failures. Therefore, for measurements of ITD sensitivity at higher pulse rates, we used an offline artifact rejection method based on template subtraction (Buechel et al., 2018) to ensure that all spikes were detected. Additional filtering $(500-3000 \mathrm{~Hz})$ was applied after artifact rejection for spike detection.

Stimuli

All stimuli were trains of biphasic pulses $(50 \mu \mathrm{s} /$ phase, no interphase gap) delivered to each CI through a pair of custom-built, high-bandwidth, isolated current sources. Current was passed between the most apical and the most basal electrode sites to excite neurons over a wide range of tonotopic axis. The search stimulus was a sequence of three pulses presented successively to both ears (diotically), the left ear and the right ear with a 100 -ms interval between each pulse and a 200 -ms silent interval between the triplets. Upon isolating a single unit, spike activity was measured as a function of current level using the search stimulus to determine the response threshold and the dynamic range. The spontaneous firing rate was measured by recording spike activity during 30 -s of silence. All units showing a driven response to the search stimulus, whether excitatory or suppressive, were further studied and included in the data set.

To characterize neural temporal coding, responses to diotic pulse trains with pulse rates ranging from 20 to $1280 \mathrm{pps}$ in half-octave steps were measured with 12 repetitions each. Stimuli were usually presented at $1-6 \mathrm{~dB}$ above the threshold of synchronized responses to a single diotic pulse.

ITD sensitivity was characterized by acquiring responses while varying the relative timing of pulse trains delivered to the two implants with zero ILD. We first varied ITD of 80-pps pulse trains from -1500 to $+1500 \mu$ s in $300-\mu$ s steps at three to four current levels within the dynamic range of the unit. The current level yielding the strongest ITD sensitivity was selected to further investigate the effect of varying pulse rate. We then varied pulse rate from 20 to $640 \mathrm{pps}$ while also varying ITD from -2000 to $+2000 \mu$ s in $200-\mu$ s steps to characterize ITD sensitivity over a wide range of pulse rates. All stimulus conditions were randomly interleaved, with 10 repetitions of each ITD/pulse rate combination. Each stimulus had a duration of $300 \mathrm{~ms}$ with a silent interval of $300 \mathrm{~ms}$ between stimuli, resulting in a repetition period of $600 \mathrm{~ms}$.

\section{Histologic processing}

\section{Electrode track reconstruction}

In three ED-CS rabbits, we made electrolytic lesions during the last recording session while the animal was under anesthesia $(6 \mathrm{mg} / \mathrm{kg}$ xylazine, s.c.; $44 \mathrm{mg} / \mathrm{kg}$ ketamine, i.m.). Electrolytic lesions were made by passing radio frequency current for $60 \mathrm{~s}$ using a lesion maker (Model LM-3, Grass Instruments) to mark the borders of the region showing evoked activity with CI stimulation. The rabbit was then perfused intracardially using a $4 \%$ paraformaldehyde (PFA) solution in PBS. The brain was immersed in fixative for $48 \mathrm{~h}$ and then transferred to $25 \%$ sucrose solution for several days. The brain was embedded in optimal cutting temperature (OCT) compound and sagittal sections $(80 \mu \mathrm{m})$ were cut with a cryostat at $-15^{\circ} \mathrm{C}$. Sections were mounted on gel-subbed slides and dehydrated with ethanol baths of increasing concentration up to $100 \%$. Cell bodies were stained with azure-thionin. Electrode tracks reaching the central nucleus of the IC through the occipital cortex and superior colliculus were identified. All lesions were located in the central nucleus of the IC (Fig. 2B).

Temporal bone processing

Both cochleas from the four ED-CS rabbits were harvested following intra-cardiac perfusion. We also harvested both cochleas from two littermates: one normal control and one early-deafened but unimplanted rabbit. Methods for temporal bone processing and stereological analysis for quantification of SGNs were the same as described previously (Chung et al., 2019). The cochleas were immersed in fixative for $24 \mathrm{~h}$ then moved to $120 \mathrm{~mm}$ EDTA and $1 \%$ glutaraldehyde ( $\mathrm{pH} 7$ ) for six to eight weeks for decalcification. Temporal bones were embedded in Araldite (Electron Microscopy Sciences) following dehydration with increasing concentrations of ethanol. Embedded temporal bones were sectioned at a thickness of $20 \mu \mathrm{m}$ and every tenth section was stained with toluidine blue (1:9 water diluted EpoxyTissue Stain; Electron Microscopy Sciences) at $60^{\circ} \mathrm{C}$ for $\sim 48 \mathrm{~h}$ then mounted on glass slides.

Stereological analysis was performed by two observers blinded to the animals' age and history of procedures with Stereo Investigator software (MicroBrightField Bioscience). A contour of Rosenthal's canal was drawn, then the sampling grid was placed over the region of interest and sampling sites were selected randomly by the software. The nucleoli of SGNs were marked if they came into focus in the sampling grid (Ishiyama et al., 2011). After systemic sampling of all stained sections, the total number of SGNs was calculated by the software.

\section{Statistical analysis}

Neural ITD sensitivity

ITD tuning curves were obtained by averaging the firing rates for each ITD over the entire stimulus duration $(0-300 \mathrm{~ms})$ and across all stimulus presentations. Neural sensitivity to ITD was quantified by the ITD signal-to-variance ratio (STVR; Hancock et al., 2010; Chung et al., 2016). ITD STVR is an ANOVA-based metric that represents the fraction of variance in firing rates because of changes in ITD relative to the total variance in firing rate across both stimulus trials and ITDs. Responses were considered to be ITD sensitive when the STVR was significantly greater than zero ( $F$ test, $p<0.01$ ). The degrees of freedom for the $F$ test were 20,189 for most cases when 21 ITD values were tested over 10 repetitions.

The ITD tuning curves were fitted to a template consisting of the sum of a Gaussian and a sigmoid function to classify the shape (Chung et al., 2016, 2019):

$$
\mathrm{R}(\mathrm{ITD})=A \cdot 2^{-\left(\frac{I \mathrm{~T} D-B}{\frac{C}{2}}\right)^{2}}+\frac{D}{1+3^{-2\left(\frac{I T D-B}{C}\right)}}+E,
$$

where $\mathrm{A}, \mathrm{D}$, and $\mathrm{E}$ are scaling factors, B is the center of both Gaussian and the sigmoid functions, and $\mathrm{C}$ represents the half-width of the Gaussian and the half-rise of the sigmoid function. The fitted curves were classified into four types: monotonic, peak, trough, and unclassified (ITD curves that were poorly fit by the template, $r^{2}<0.75$ ). From the fitted curves, the best ITD (ITD best, the ITD at the maximum of peak shaped responses), and $\mathrm{ITD}_{\mathrm{MS}}$, the ITD at the maximum slope, were derived. Only ITD tuning curves in response to pulse rates below 160 pps were used in shape classification and to calculate of ITD metrics to avoid multiple cycles of periodicity within the measured ITD range of $\pm 2000 \mu$ s.

\section{Excitatory and suppressive responses}

To quantify the prevalence of excitatory and suppressive response in each neuron, the sustained firing rate during the pulse train presentation ("on-period") was compared with the sustained firing rate during the silent interval between the stimuli ("off-period"). The first $30 \mathrm{~ms}$ of the 300-ms stimulus were excluded to separate the sustained, on-period response from the onset response, and likewise, the first $100 \mathrm{~ms}$ of the 300 -ms interstimulus period was excluded to separate the sustained, offperiod response from any rebound response.

The prevalence of sustained excitatory and suppressive responses was quantified by two methods for each unit: (1) comparing the firing rates during the on-period and off-period on a trial-by-trial basis for each pulse rate and (2) defining an excitatory/suppressive (E/S) index to quantify the relative prevalence of excitatory and suppressive activity across all pulse rates (Chung et al., 2019). A response to a given pulse rate was considered excitatory if the mean on-period firing rate was significantly higher (paired $t$ test, two-tailed, $p<0.01$ ) than the mean offperiod firing rate. Conversely, the response was considered suppressive if the mean on-period firing rate was significantly lower than the mean off-period rate. The E/S index was obtained by comparing the excitatory area $\mathrm{E}$ to the suppressive area $\mathrm{S}$. The excitatory area $\mathrm{E}$ was defined as the 
sum of the on-period firing rates minus the off-period firing rates over all pulse rates that evoked a significant excitatory response. Similarly, the suppressive area $S$ was defined as the sum of the off-period firing rate minus the on-period firing rate over the pulse rates that evoked a significant suppressive response. The $\mathrm{E} / \mathrm{S}$ index is the ratio $(\mathrm{E}-\mathrm{S}) /(\mathrm{E}+\mathrm{S})$ that ranges from -1 for a purely suppressive response to +1 for a purely excitatory response.

\section{Synchronized response to pulse trains}

The prevalence of synchronized responses and the highest pulse rate that elicited synchronized response were compared across animal groups. To quantify the synchrony of spikes to individual pulses, the stimulus pulse train was cross-correlated with the spike trains (Hancock et al., 2012; Chung et al., 2014, 2019). The 30-ms onset response was excluded from the spike train to avoid biasing the synchrony estimate by prominent peaks at the onset. To assess the statistical significance of cross-correlation peaks, the cross-correlation was computed for 5000 random spike trains containing the same number of spikes as the original neural recording. A confidence bound for the null hypothesis of no correlation was defined as the 99.5th percentile of the cross-correlograms across the 5000 random spike trains. A peak in the cross-correlogram was considered significant when it exceeded the confidence bound. The upper rate limit of synchronized responses was computed by linearly interpolating the height of the cross-correlogram peaks to find the pulse rate where the peak height intercepted the confidence bound.

\section{Statistical methods for group analysis}

To compare the fractions of neurons showing significant ITD sensitivity, E/S responses, and significant synchronized responses as a function of pulse rate across the three animal groups, two-way ANOVAs were run on the arcsine-transformed fractions. For categorical data such as distributions of ITD tuning shapes, $\chi^{2}$ tests were used. A Kruskal-Wallis one-way ANOVA, a non-parametric test, was used to compare the distributions of E/S index, spontaneous firing rates, upper limit of synchronization, and SGN counts between the three animal groups. One-way ANOVAs were used to compare the mean $\mathrm{ITD}_{\text {best }}$ and $\mathrm{ITD}_{\mathrm{MS}}$ across the three animal groups. The Kendall rank correlation coefficient was used to test for a longitudinal effect of deafness duration on SGN counts and a correlation between SGN counts and ITD STVR across animals. Lastly, a binomial test was used to test whether the rabbits performed better than chance level in the behavioral task.

\section{Results}

We recorded from 132 single units in the IC of four rabbits that were neonatally deafened and provided both chronic stimulation with bilateral CIs and behavioral training in a spatial hearing task starting at two months of age (ED-CS). Neural ITD sensitivity and temporal coding were compared with previously reported data from 117 neurons in three neonatally deafened and unstimulated rabbits (ED-US; Chung et al., 2019) and 180 neurons from four rabbits that were deafened as adults (AD; Chung et al., 2016; Fig. 1). The last two groups of animals only received electric simulation during the 2 -h electrophysiological recording sessions.

\section{Behavioral training}

Figure 3 shows performance in the behavioral task as a function of session number for all four rabbits. Because the number of potential targets varied across sessions and animals, performance is quantified by the $p$ value for a binominal test comparing the fraction of correctly identified targets to chance in each session. All four rabbits learned to locate the target using combined auditory and visual cues (i.e., $p<0.05$ ) in $10-20$ sessions. However, none learned to locate the target based only on auditory cues after being weaned off the visual cues. In two animals (J6 and J7), behavioral training was discontinued after 10-20 sessions in which auditory only and combined auditory and visual trials were both presented. In two animals (J8 and J10), behavioral training with primarily auditory only trials were continued for $\sim 15$ more sessions. These animals did not show any improvement in performance although they still performed better than chance accuracy when occasionally tested with audiovisual cues.

In the absence of comparable behavioral data from $\mathrm{NH}$ and $\mathrm{AD}$ rabbits, it is not possible to pinpoint the reason behind the failure of the ED-CS animals to learn the localization task. This failure might be because of one or more of the following reasons: (1) binaural sensitivity in juvenile rabbits at two to four months of age is not mature in general; (2) binaural circuitry in the ED$\mathrm{CS}$ rabbits that started receiving CI stimulation at two months of age require auditory experience over a longer period of time or more exposure in each session to develop; (3) binaural cues provided by bilateral CI stimulation are not accessible to the animals or not sufficiently salient. In addition, because the ED-CS rabbits received both passive stimulation with environment sounds and behavioral training, it is not possible to ascertain which of these two interventions is the primary driver of the differences in ITD sensitivity we observed between ED-CS and ED-US rabbits. In contrast to the current study, Isaiah et al. (2014) showed that neonatally-deafened and adult-implanted ferrets were able to learn to localize sound with training on an audiovisual task involving interleaved presentation of auditory and visual stimuli, with corresponding improvements occurring in ILD sensitivity in auditory cortex.

\section{Restoration of ITD sensitivity in early-deaf animals with bilateral CI stimulation}

ITD sensitivity of single IC neurons was characterized by measuring responses to periodic pulse trains with ITDs ranging from -2000 to $+2000 \mu \mathrm{s}$ and pulse rates from 20 to $640 \mathrm{pps}$. Figure $4 A-D$ shows temporal response patterns to 160 -pps pulse trains with varying ITDs obtained in example neurons from an ED-US rabbit, an AD rabbit, and two ED-CS rabbits. The neuron from an ED-US rabbit showed responses primarily at stimulus onset (Fig. 4A). This neuron's ITD tuning curve (Fig. 4E) did not show significant ITD sensitivity based on the ITD STVR. In contrast, the neuron from an ED-CS rabbit shown in Figure $4 C$ had a strong onset response for contralateral-leading $(>0)$ ITDs and also a sustained response for ITDs of $0-400 \mu \mathrm{s}$. Its ITD tuning curve was peak-shaped (Fig. $4 E$ ) and the ITD sensitivity was significant based on the ITD STVR. Figure $4 D$ shows results for a neuron from another ED-CS rabbit that showed weak but significant contralateral-preferring ITD tuning. The neuron from an $\mathrm{AD}$ rabbit also showed contralateral-preferring ITD tuning, with sustained firing near $400 \mu$ s (Fig. $4 B$ ) and significant ITD sensitivity (Fig. 4E).

The ITD sensitivity of IC neurons to bilateral CI stimulation is known to degrade with increasing pulse rate, consistent with the limitations in perceptual ITD sensitivity in human CI listeners (Smith and Delgutte, 2007; Chung et al., 2016). This rate-dependent degradation in ITD sensitivity is more severe in ED-US animals than in AD rabbits (Chung et al., 2019). We compared the dependence of ITD sensitivity on pulse rate to investigate whether chronic CI stimulation during development can increase the range of pulse rates over which IC neurons are ITD sensitive. Figure $5 A-D$ shows ITD tuning curves of the same example units as in Figure 4 for different pulse rates. The neuron from an EDUS rabbit (Fig. 5A) showed a weak preference to contralateralleading ITDs at low pulse rates (40-80 pps) but little or no ITD sensitivity at higher pulse rates. In contrast, the neuron from an 


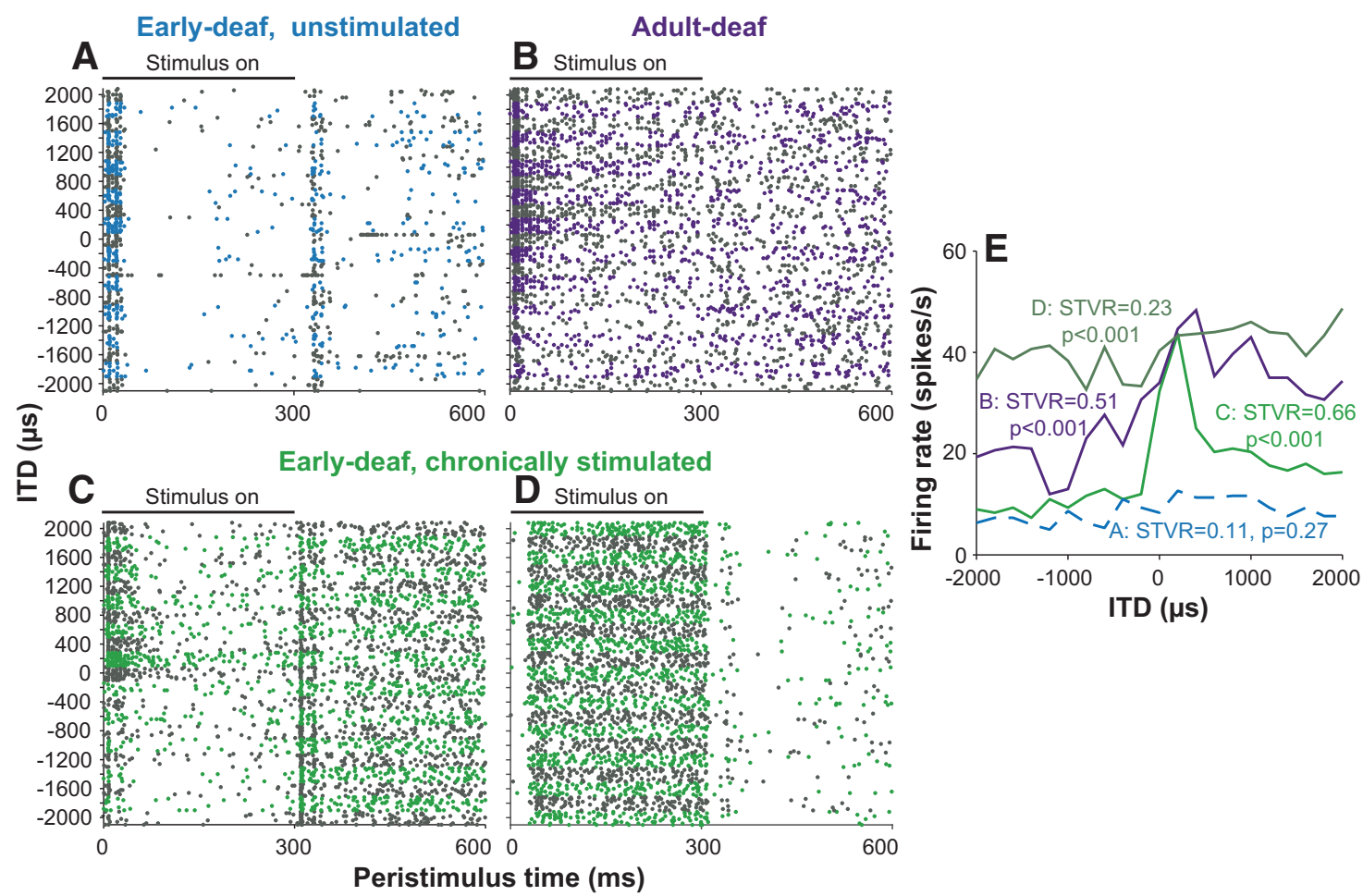

Figure 4. Temporal discharge pattern (dot rasters) to 160-pps pulse trains as a function of ITD for example neurons from an ED-US rabbit (A), an AD rabbit (B), and two neurons from ED-CS rabbits $(\boldsymbol{C}, \boldsymbol{D})$. One neuron $(\boldsymbol{C})$ shows sharp ITD tuning while the other one $(\boldsymbol{D})$ shows more typical ITD sensitivity. Alternating colors indicate blocks of stimulus trials at different ITDs. $\boldsymbol{E}$, Firing rate versus ITD curves for the four example neurons. Solid lines indicate statistically significant ITD sensitivity based on ANOVA $(p<0.01)$.
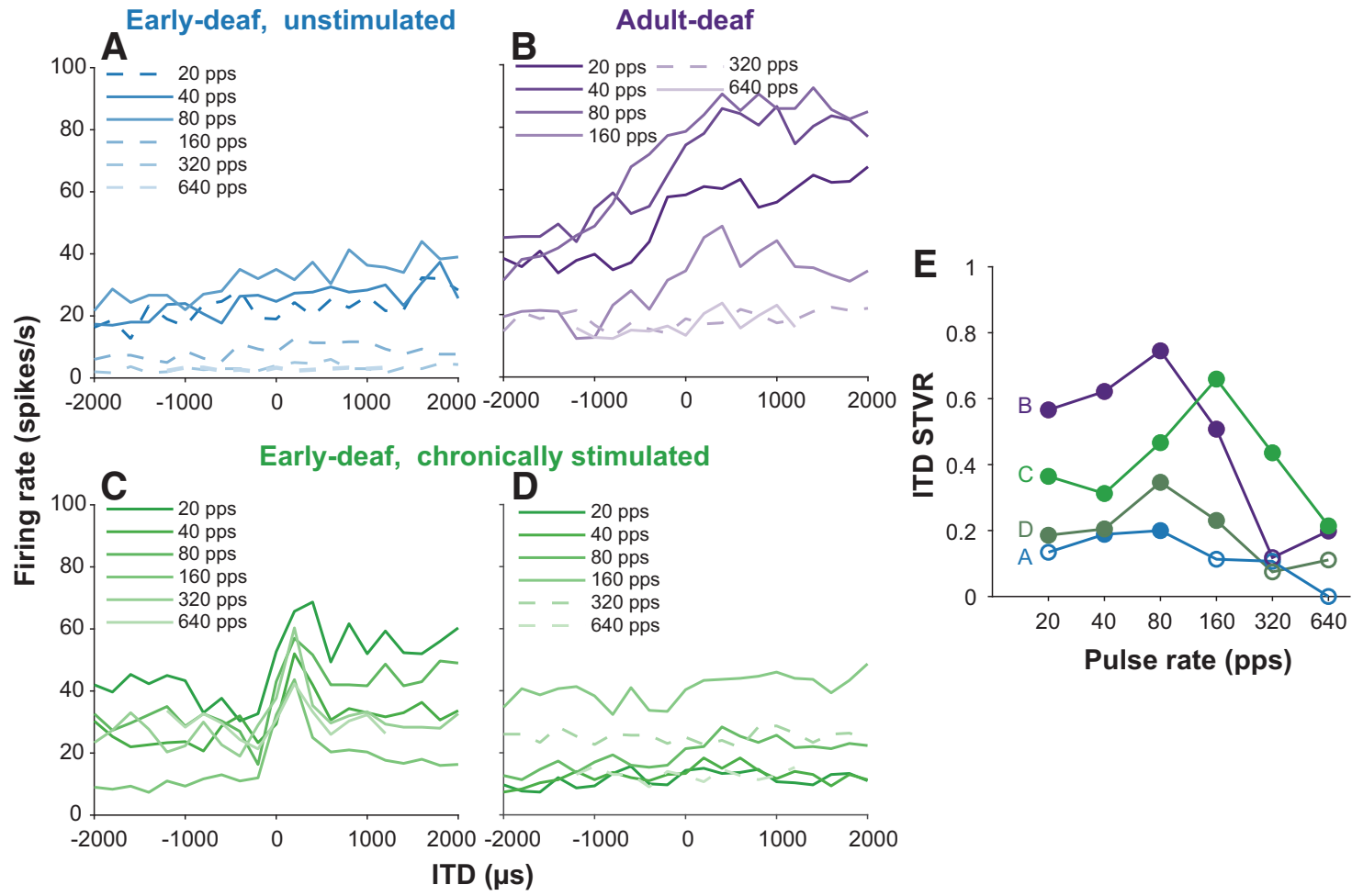

Figure 5. $A-D$, Firing rate versus ITD for a range of pulse rates for the same four example neurons as in Figure 4. E, ITD STVR as a function of pulse rate for the four example neurons. Solid lines $(\boldsymbol{A}-\boldsymbol{D})$ and filled circles $(\boldsymbol{E})$ indicate statistically significant ITD STVR $(\boldsymbol{p}<0.01)$. 
ED-CS rabbit shown in Figure $5 C$ showed sharper ITD tuning, with a peak near $400 \mu$ s for all pulse rates tested. The second example ED-CS neuron (Fig. 5D) showed weak ITD tuning with a slight contralateral preference that was significant over 20-160 pps. The neuron from an AD rabbit showed clear ITD tuning, with a preference for contralateral-leading ITDs (Fig. 5B). The ITD STVR (Fig. 5E) was statistically significant over a wider range of pulse rates in both $\mathrm{ED}$-CS neurons and in the $\mathrm{AD}$ neuron than in the ED-US neuron, where it was limited to a narrow range of pulse rates (40-80 pps).

The restoration of ITD sensitivity over a wide range of pulse rates seen in the example ED-CS neurons of Figure 5 is representative of the corresponding neural populations. For all pulse rates tested, more neurons from the ED-CS group were sensitive to ITD, as measured by the STVR, compared with the ED-US group (Fig. 6A). The fraction of ITD sensitive neurons in the $\mathrm{ED}-\mathrm{CS}$ group was comparable to the fraction in the AD group at most rates. A two-way ANOVA on the arcsine-transformed fractions of ITD sensitive units showed significant effects of both pulse rate $\left(F_{(5,17)}=25.49, p<0.001\right)$ and animal group $\left(F_{(2,17)}=25.08, p<0.001\right)$. (Only units that were tested for at least two pulse rates were included in this analysis.) Post hoc paired comparisons (Tukey-Kramer) showed that the fraction of ITD sensitive neurons was significantly lower in the ED-US group compared with the each of the other two groups (ED-CS: $p=0.0006$, $\mathrm{AD}: p=0.0002)$, whereas there was no difference between the ED-CS and AD groups $(p=0.5751)$.

The median ITD STVR (Fig. $6 B$ ) was higher for the EDCS group than for the ED-US group at most pulse rates $(>20$ pps), but lower for all pulse rates tested compared with the AD group. The largest effect of chronic stimulation during development was seen for pulse rates above 80 pps. A twoway ANOVA on the arcsine-transformed STVRs showed significant effects of both pulse rate $\left(F_{(5,1304)}=20.72, p<0.001\right)$ with medium effect size $\left(\omega_{p}{ }^{2}=0.070\right)$ and animal group $\left(F_{(2,1304)}=14.27, p<0.001\right)$ with small effect size $\left(\omega_{p}{ }^{2}=\right.$ $0.020)$ but no interaction $\left(F_{(10,1304)}=0.6, p=0.81\right.$; for the magnitude of effect size, see Cohen, 1988). Post hoc paired comparisons (Tukey-Kramer) showed that the ITD STVR was significantly higher in the ED-CS group compared with the ED-US group $(p=0.0032)$, but significantly lower compared with the AD group ( $p=0.0098)$.

We tested the relative contribution of onset and sustained response across animal groups and pulse rates by repeating the above analysis on two separate time segments. We found that fewer neurons were ITD sensitive based on the first $30 \mathrm{~ms}$ of the response ("onset") compared with fractions based on the remaining $270 \mathrm{~ms}$ of the response ("sustained"; three-way ANOVA, $\left.F_{(1,35)}=7.32, p=0.012\right)$. There was no significant difference between the onset ITD STVR compared with sustained ITD STVR (three-way ANOVA, $\left.F_{(1,2609)}=0.05, p<0.83\right)$, although there was a significant interactions between analysis segment and pulse rate $\left(F_{(5,2609)}=7.93, p<0.001\right)$ and animal group $\left(F_{(2,2609)}=6.00\right.$, $p=0.0025)$. Post hoc comparisons (Tukey-Kramer) showed that there were no differences in onset STVR between groups whereas sustained STVR showed significant differences in the same order as reported based on all responses. Similarly, sustained STVR was more dependent on the pulse rate than onset STVR similar to the findings in acutely deafened, anesthetized cats (Smith and Delgutte, 2007).

To compare the shapes of ITD tuning curves from the three groups of rabbits, the tuning curves were fit to a flexible template
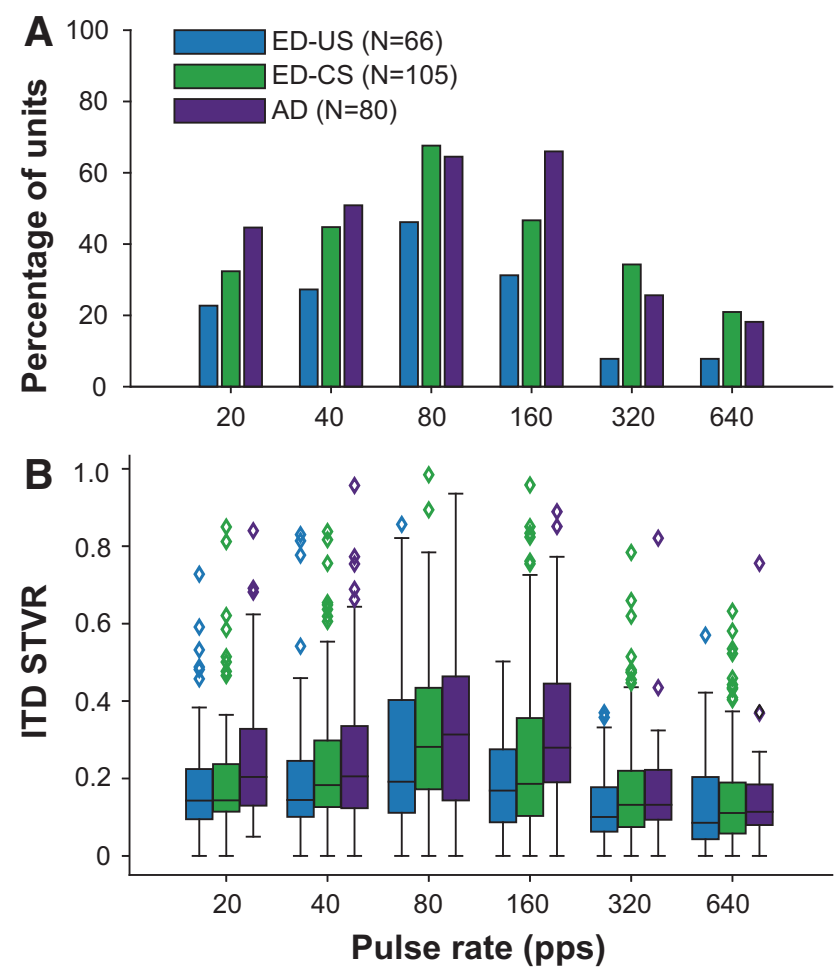

Figure 6. $A$, Fraction of ITD-sensitive neurons as a function of pulse rate for early-deaf and unstimulated, early-deaf and stimulated, and AD animals. $\boldsymbol{B}$, ITD STVR versus pulse rate for the three animal groups. Horizontal bars show medians, the interquartile range (IQR) is given by box size; whiskers extend to the most extreme data points within $\pm 1.5 \times I Q R$.

that was used to classify the shapes into four categories: monotonic, peak, trough, and other (unclassified). Only units that showed significant ITD sensitivity based on the STVR for at least one pulse rate below 160 pps were used for the shape classification. For units that showed significant ITD sensitivity at multiple pulse rates, the ITD tuning curve with the highest STVR was used for shape classification. The relative incidences of the four shapes were similar in all three animal groups (Fig. 7A) although there were slightly more unclassified types in both ED groups than in the $\mathrm{AD}$ group $\left[\chi^{2}(6\right.$, $N=229)=2.4605, p=0.8729$ ].

Two ITD metrics were calculated from the fitted curve to further characterize how ITD tuning characteristics depend on the animal group: the best ITD, derived from peak-shaped responses, and $\mathrm{ITD}_{\mathrm{MS}}$, the ITD at the maximum slope. Figure $7 B$ compares the distributions of best ITDs between the three animal groups. $\mathrm{ITD}_{\text {best }}$ in the ED-CS group showed a statistically significant contralateral bias (two-tailed $t$ test, mean $=277 \mu \mathrm{s}$, $\left.t_{(55)}=5.6837, p<0.001\right)$. Such a contralateral bias in ITD $_{\text {best }}$ was also observed in the AD group (two-tailed $t$ test, mean $=162 \mu \mathrm{s}$, $t_{(52)}=3.4852, p=0.0010$ ), but not in the ED-US group (twotailed $t$ test, mean $\left.=92 \mu \mathrm{s}, t_{(22)}=0.9316, p=0.3616\right)$. However, the differences in mean ITD $_{\text {best }}$ between the three animal groups did not reach statistical significance (ANOVA, $F_{(2,131)}=2.39$, $p=0.0953$ ). ITD $_{\mathrm{MS}}$ (Fig. 7C) was broadly distributed with a mean near zero for all three groups (ED-CS: $-53.4 \mu \mathrm{s}, \mathrm{AD}$ : $-78.3 \mu \mathrm{s}$, ED-US: $8.2 \mu \mathrm{s}$ ) and there were no statistically significant differences in $\mathrm{ITD}_{\mathrm{MS}}$ between the three animal groups (ANOVA, $\left.F_{(2,262)}=0.32, p=0.7261\right)$.

In summary, there was a minimal effect of different auditory experience during development on the distribution of ITD tuning shapes or the ITD tuning metrics, except for the presence of 

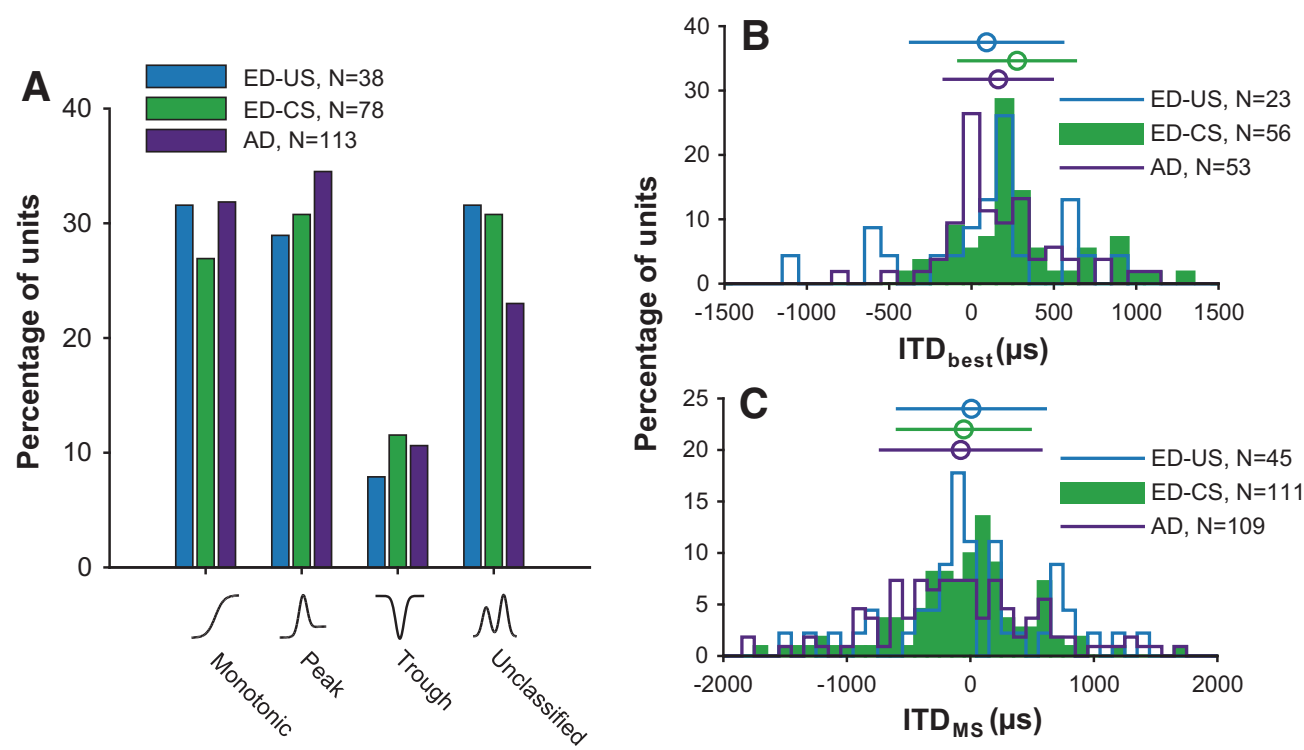

Figure 7. Distribution of ITD tuning shapes $(\boldsymbol{A})$, best ITDs $(\boldsymbol{B})$, and ITD $\mathrm{MS}(\boldsymbol{C})$ for the three animal groups. Error bars above each histogram represent means \pm 1 SD.

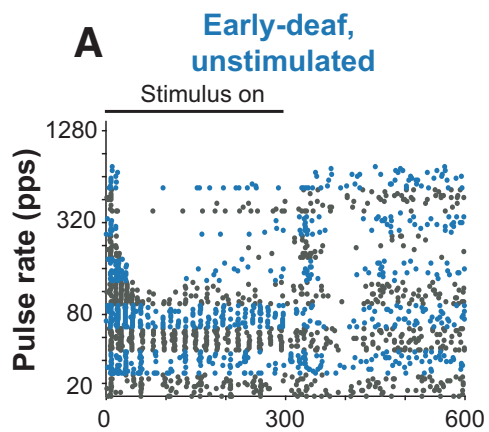

B Adult-deaf

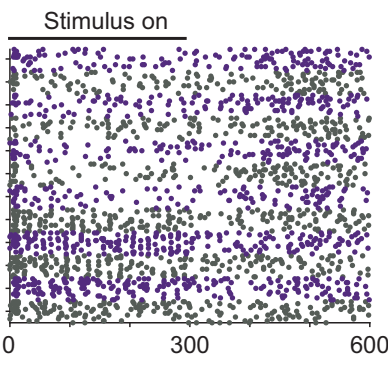

Peristimulus time (ms)

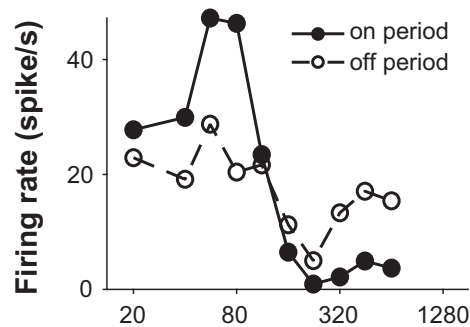

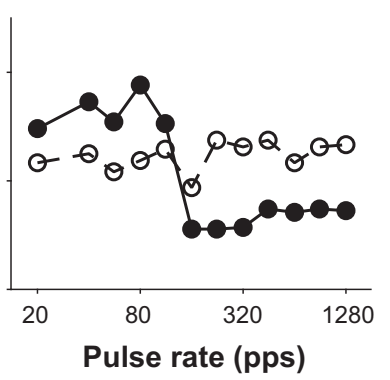

C Early-deaf, chronically stimulated Stimulus on
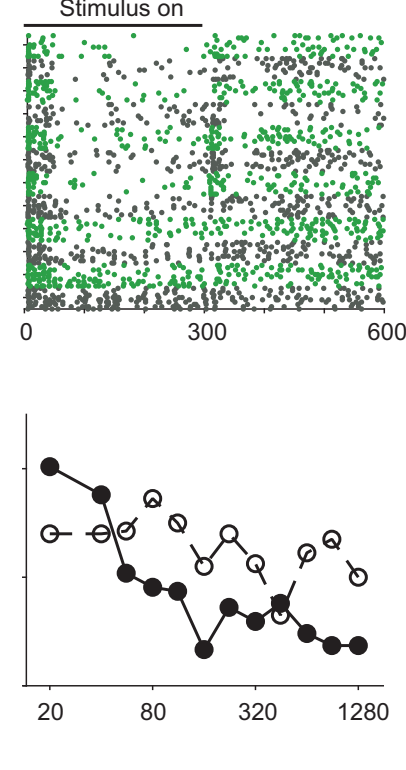

Figure 8. A-C, Top, Temporal discharge patterns (dot rasters) as a function of pulse rate for the same three example neurons as in Figures $4,5 A-C$. Alternating colors indicate blocks of stimulus trials at different pulse rates. Bottom, Mean sustained firing rate as a function of pulse rate during stimulus on-period and off-period for the three example neurons.

a contralateral bias in $\mathrm{ITD}_{\text {best }}$ in the ED-CS and AD groups, but not the ED-US group.

\section{Chronic CI stimulation did not restore excitatory responses in early-deaf animals}

To characterize how the temporal response pattern to electrical stimulation depends on auditory experience, we measured responses to diotic electric pulse trains using finer steps of pulse rate than in the ITD measurements described so far. In all three animal groups, there was substantial variability in the pulserate dependence of firing rates and the relationship between responses during the on-period and off-period. Temporal response patterns and profiles of firing rate versus pulse rate from the three example neurons shown in Figures 4, 5A-C are shown in Figure 8. The neuron from an ED-US rabbit (Fig. $8 \mathrm{~A}$ ) showed a sustained and synchronized firing pattern up to $80 \mathrm{pps}$, with higher firing rates during the on-period than during the off-period (excitatory response). For pulse rates above $80 \mathrm{pps}$, the excitatory response only lasted for $\sim 20 \mathrm{~ms}$ after the stimulus onset and was followed by a suppressive response for pulse rates above $224 \mathrm{pps}$. The neuron from an $\mathrm{AD}$ rabbit (Fig. $8 B$ ) showed sustained, synchronized responses up to 112 pps. This neuron also showed small suppression of sustained firing rates for pulse rates above 112 pps. Lastly the neuron from an ED-CS rabbit (Fig. 8C) showed poorly synchronized responses only lasting for 50$60 \mathrm{~ms}$ after stimulus onset followed by a suppressive response for pulse rates above $40 \mathrm{pps}$. 

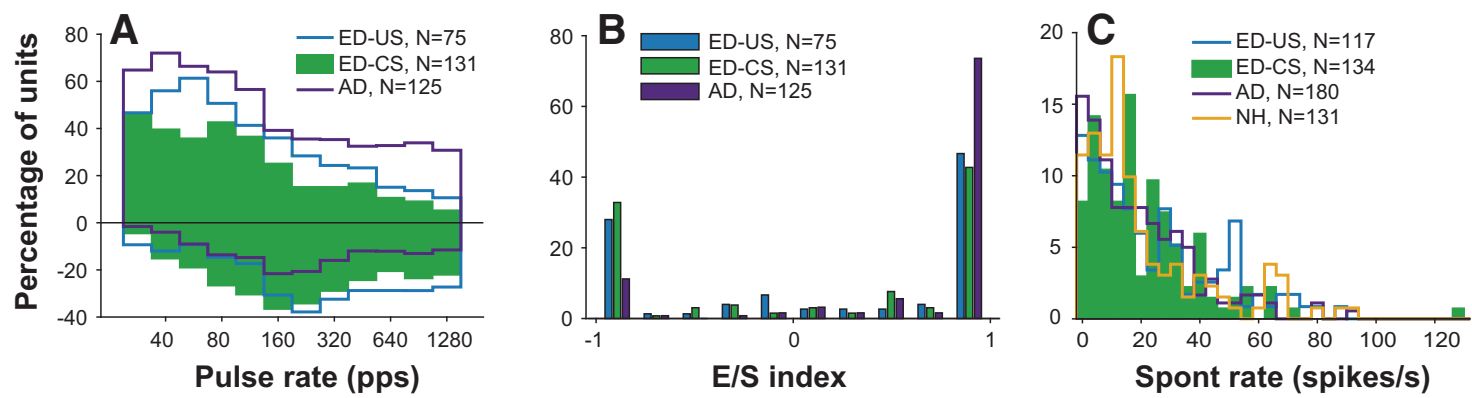

Figure 9. A, Percentage of units showing significant excitatory (positive ordinates) and suppressive (negative ordinates) response to pulse train stimulation as a function of pulse rate for the three animal groups. $\boldsymbol{B}$, Distribution of $\mathrm{E} / \mathrm{S}$ ratio for the animal groups. $\boldsymbol{C}$, Distribution of spontaneous firing rates in the three animal groups and $\mathrm{NH}$ animals.

For each pulse rate tested, the firing rate during the on-period (excluding the first $30 \mathrm{~ms}$ ) was compared on a trial-by-trial basis to the rate during the off-period (excluding the first $100 \mathrm{~ms}$ ) to determine whether the stimulus evoked significant sustained excitatory or suppressive activity. Figure $9 A$ shows the fractions of IC neurons showing excitatory and suppressive responses to pulse train stimuli as a function of pulse rate for the three animal groups. Positive ordinates represent excitatory responses and negative ordinates indicate suppressive responses. There were significant effects of both pulse rate $\left(F_{(11,35)}=38.95, p<0.001\right)$ and animal group $\left(F_{(2,35)}=81.11, p<0.001\right)$, as revealed by a two-way ANOVA on the arcsine-transformed fraction of excitatory responses. Previously, we reported a reduction in excitatory activity in the ED-US group compared with the $\mathrm{AD}$ group (Chung et al., 2019). Unexpectedly, we found a further reduction in excitatory activity in the ED-CS group compared with the EDUS group (post hoc paired comparisons with Tukey-Kramer correction, $p<0.001$ ). Figure $9 A$ also shows an increase in suppressive activity in both $\mathrm{ED}$ groups compared with the $\mathrm{AD}$ group. A two-way ANOVA on the arcsine-transformed fraction of suppressive responses showed significant effects of both pulse rate $\left(F_{(11,35)}=12.08, p<0.001\right)$ and animal groups $\left(F_{(2,35)}=35.49\right.$, $p<0.001$ ). Post hoc paired comparisons (Tukey-Kramer) confirmed that the fraction of suppressive responses was significantly lower in the AD group compared with both $\mathrm{ED}$ groups $(p<0.001$ for both) but there was no difference between the two ED groups $(p=0.8717)$.

We defined an E/S ratio for each unit to characterize the relative prevalence of excitatory versus suppressive responses across pulse rates in the three animal groups (Chung et al., 2019; Fig. 9B). The E/S ratio ranges from -1 for a purely suppressive response at all pulse rates to +1 for a purely excitatory response. A Kruskal-Wallis test showed a significant effect of animal group on the $\mathrm{E} / \mathrm{S}$ ratio $\left[\chi^{2}(2,330)=18.82\right.$, $p<0.001]$. Post hoc paired comparisons (Tukey-Kramer) showed that the median $\mathrm{E} / \mathrm{S}$ ratio was larger in the $\mathrm{AD}$ group compared with both ED groups (ED-US: $p=0.0198$, ED-CD: $p=0.0001)$ but there was no difference between the two ED groups $(p=0.6073)$. Consistent with the findings about the fractions of excitatory and suppressive responses, chronic stimulation did not eliminate the reduction in $\mathrm{E} / \mathrm{S}$ ratio observed in early-onset deafness.

The differences in relative fractions of E/S responses between animal groups might be driven by differences in spontaneous activity of the IC neurons. We have previously shown that there was no significant difference in spontaneous firing rates (SR) between $\mathrm{AD}$, ED-US, and $\mathrm{NH}$ animals (Chung et al., 2019). Figure 9C compares the SR distribution in the three animal groups as well as in NH rabbits. Consistent with the previous results, the median SR in the ED-CS group (16.6 spikes/s) lay in a similar range as in the other animal groups (ED-US $=16.4$ spikes $/ \mathrm{s}, \mathrm{AD}=15.1 \mathrm{spikes} / \mathrm{s}$, $\mathrm{NH}=12.6$ spikes/s) and did not differ statistically [Kruskal-Wallis test, $\chi^{2}(3,561)=3.73, p=0.2919$ ]. In general, we did not find an effect of deafness and auditory experience on spontaneous activity.

\section{Chronic CI stimulation did not improve temporal coding in early-deaf animals}

We next examined whether there was an effect of chronic bilateral CI stimulation on synchronized responses to pulse train stimuli. For each neuron and pulse rate, the spike train was cross-correlated to the stimulus to quantify the neural synchrony and define the upper pulse rate limit of synchronized activity (Hancock et al., 2012; Chung et al., 2014, 2019). Figure 10A compares the fractions of neurons that showed significant synchronized responses as a function of pulse rate in the three animal groups. For all pulse rates, the $\mathrm{AD}$ group showed the largest fraction of synchronized responses compared with both ED groups. Similarly as the fraction of excitatory responses, the fraction of synchronized responses was smaller in the ED-CS group than in the ED-US group for all rates tested. A two-way ANOVA on arcsine-transformed fractions showed significant effects of both animal group $\left(F_{(2,35)}=29.81, p<0.001\right)$ and pulse rate $\left(F_{(11,35)}=\right.$ 107.12 , $p<0.001$ ). Post hoc paired comparisons (TukeyKramer) showed that the fraction of synchronized responses in the ED-US group was smaller than in the AD group $(p=0.0033)$ but larger than in the ED-CS group $(p=0.0024)$. Figure $10 B$ compares the cumulative distributions of upper limits of synchronized response in three animal groups. In this analysis, neurons that did not synchronize to any pulse rate were excluded. The cumulative distributions from three animal groups largely overlapped with each other and did not differ statistically [Kruskal-Wallis test, $\chi^{2}(2,256)=3.25, p=0.1973$ ] .

In summary, chronic CI stimulation did not improve temporal coding in early deaf animals. Fewer neurons in the ED-CS group showed sustained excitatory responses and synchronized responses to pulse train stimulation compared with the ED-US group. Therefore, the restoration of response properties with bilateral CI stimulation was limited to ITD sensitivity.

\section{Count of SGNs}

The differences in ITD sensitivity and temporal coding observed between the three animal groups could be driven by differences in survival of SGNs. To test for this possibility, total SGN counts were estimated by the optical fractionator technique in both ears of all four ED-CS rabbits and compared with SGN counts from ED-US rabbits and $\mathrm{NH}$ rabbits, including one coming from the 
A

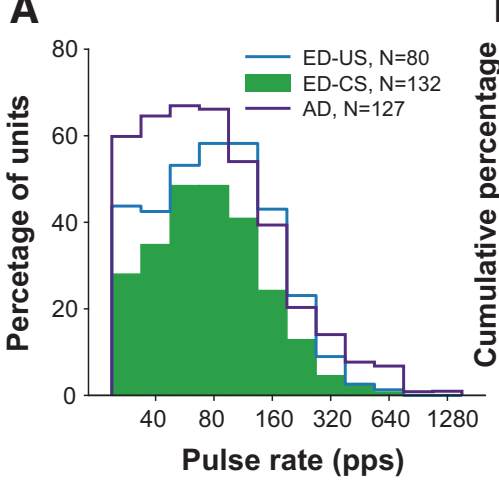

B

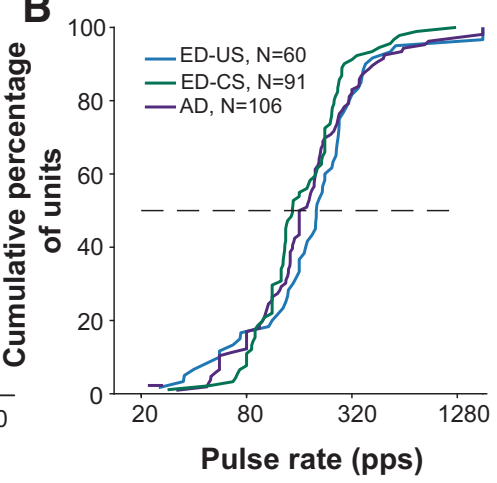

Figure 10. A, Percentage of units showing significant synchronized response to pulse train stimulation as a function of pulse rate for the three animal groups. $\boldsymbol{B}$, Cumulative distribution of upper limits of synchronized response for the three animal groups.

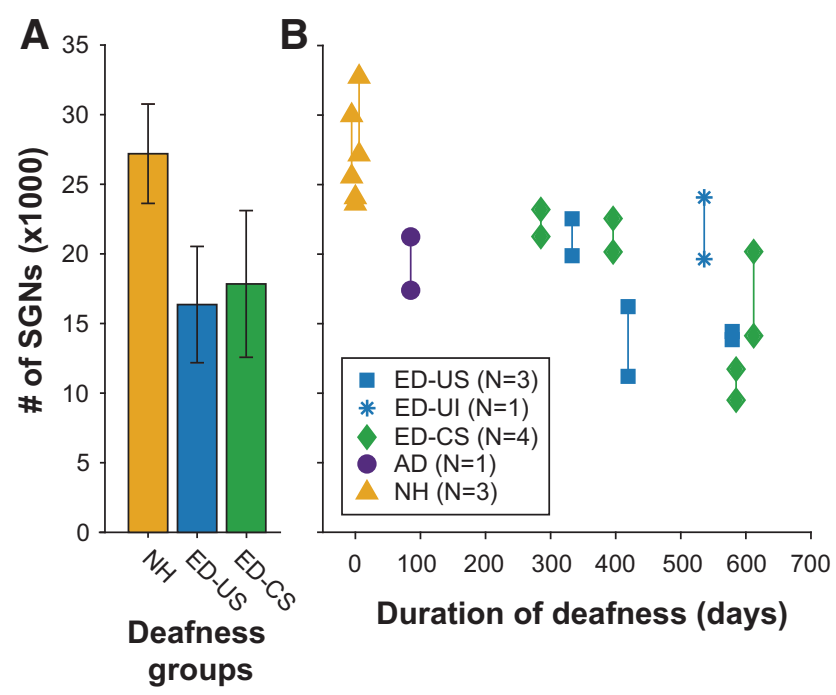

Figure 11. Estimated total SGN counts in various animal groups. $\boldsymbol{A}$, Mean SGN counts for the ED-CS and ED-US animals providing the electrophysiological data as well as three $\mathrm{NH}$ controls. Error bars represent \pm 1 SD. $\boldsymbol{B}$, Total SGN count as a function of duration of deafness in all animal groups. ED: early-deaf, -US: unstimulated, -UI: unimplanted, -CS: chronically stimulated, AD: adult-deaf, NH: normal hearing. The data points for both ears of each animal are connected by a line.

same litter as the ED-CS animals. Total SGN counts in the EDCS and ED-US groups largely overlapped with each other (Fig. $11 A$; Table 1). SGN counts in the ED-CS group ranged from $35 \%$ to $85 \%$ of the mean SGN counts from NH controls, while SGN counts in the ED-US group ranged from $41 \%$ to $83 \%$ of normal. A Kruskal-Wallis test across NH, ED-US, and ED-CS groups confirmed a significant effect of animal group on the median SGN count $\left[\chi^{2}(2,17)=12.3, p=0.0021\right]$. Post hoc paired comparisons (Tukey-Kramer) showed that SGN counts were higher in the $\mathrm{NH}$ group compared with each of the two ED groups (ED-US: $p=0.0037$, ED-CS: $p=0.0106$ ), whereas there was no difference between the ED-US and ED-CS groups $(p=0.8476)$. SGN counts from one animal that was early-deafened but not implanted (ED-UI) were in the same range as in the early-deafened and implanted groups, suggesting that the reduction in SGNs in ED-CD and ED-US animals was not because of implantation trauma. Similarly, one AD rabbit that was deafened with the same method as the AD rabbits used for electrophysiological studies and euthanized approximately

three months after deafening showed a reduction in SGNs in the same range is in ED rabbits. Based on these results from the small number of animals, it appears that deafness of sufficient duration is the primary determinant of the SGN survival rather than factors such as the age at onset of deafness, implantation trauma, or auditory experience with the implant. The substantial reduction in SGN counts observed in this AD animal is consistent with a report that SGN degeneration stabilizes after six weeks in guinea pigs (van Loon et al., 2013).

We also tested whether there was a longitudinal effect on SGN counts, i.e., whether there was a continuous decrease in SGN counts as a function of duration of deafness (Fig. 11B). There was a small but significant effect of duration of deafness when all animal groups were combined (AD, ED-US, ED-UI, and ED-CS, Kendall rank correlation coefficient, $\tau=$ $-0.3638, p=0.0433)$. This trend was stronger when only the ED-US and ED-CS groups were included (Kendall rank correlation coefficient, $\tau=-0.5033, p=0.0174)$. However, because of small sample sizes, the correlation did not reach significance when each group was tested separately (ED-US: $\rho=$ $-0.5963, p=0.1778$, ED-CS: $\tau=-0.4629, p=0.1659)$. Taken together, these results suggest it is unlikely that the observed differences in ITD sensitivity of IC neurons between the three animal groups are because of difference in survival of SGNs. We cannot rule out, however, that more subtle degenerative changes in the processes of SGNs that do not (or do not yet) impact survival of somata could contribute to the physiological differences we observed between animal groups.

Lastly, we tested whether there is a correlation between SGN counts at the end of the study and the ITD STVR within each ED-CS group. We found a small but significant positive correlation between SGN counts and ITD STVR within the ED-CS group when ITD STVR measurements from all pulse rates tested were included for each unit (Kendall rank correlation coefficient, $\tau=0.0814, p=0.0076)$. However, this trend failed to reach significance when only the maximum ITD STVR for each unit was included in the analysis (Kendall rank correlation coefficient, $\tau=$ $0.0943, p=0.2091)$. On the other hand, we also found a negative correlation in the ED-US group when measurements from all pulse rates were included (Kendall rank correlation coefficient, $\tau=-0.0875, p=0.0271)$. Overall, it is difficult to draw a firm conclusion from these results which are based on SGN counts at the end of the study from a small number of animals.

\section{Discussion}

We characterized neural ITD sensitivity and temporal coding of IC neurons in rabbits that were neonatally deafened and both behavioral trained in a spatial hearing task and chronically stimulated with bilateral CIs during development (ED-CS). The response properties were compared with data from both ED rabbits that received no stimulation during development (ED-US) and $\mathrm{AD}$ rabbits that experienced normal auditory development. We found that chronic bilateral CI stimulation during development could partly reverse the effect of auditory deprivation and restore ITD sensitivity in IC neurons. The prevalence of ITD sensitive neurons in the ED-CS rabbits was restored to a level comparable to that in $\mathrm{AD}$ rabbits. We also found an improvement in ITD sensitivity as measured by STVR in the ED-CS rabbits compared with ED-US rabbits. However, ITD sensitivity in the ED- 
$\mathrm{CS}$ rabbits was still poorer than in the $\mathrm{AD}$ rabbits. In contrast, the prevalence of excitatory responses and synchronized responses was not improved in ED-CS rabbits compared with ED-US rabbits. We did not find differences in spontaneous activity between the three groups of animals, consistent with previous results that showed no effect of auditory deprivation on SR in IC neurons in unanesthetized rabbits. Lastly, we observed a similar reduction in total SGN counts in ED-CS and ED-US animals compared with $\mathrm{NH}$ animals.

\section{Comparison with previous studies}

Previous studies in anesthetized cats have reported improved temporal coding of electric pulse train stimuli in the IC of animals that were neonatally deafened and chronically stimulated during development (Snyder et al., 1995; Vollmer et al., 1999) and also in neonatally-deafened animals that were given chronic stimulation after a long period of auditory deprivation (Vollmer et al., 2005). In contrast, the current study did not find a reversal of the effect of auditory deprivation on neural temporal coding.

This difference may stem from differences in temporal properties of the chronic CI stimulation delivered to the animals. We used the FAST strategy that is designed to deliver ITD cues and employs lower average pulse rates than the rates used in previous studies that found a restoration of temporal coding. Indeed, Vollmer et al. (1999) showed that the effect of passive stimulation on temporal coding by IC neurons depends on the temporal properties of the electrical stimulation. Neonatally-deafened cats that received chronic low-rate stimulation (30-80 pps) did not show an increase in the upper limit of synchronized response compared with unstimulated animals, while animals that received high-rate stimulation (300 pps) showed an improvement in temporal coding. The lack of improvement in neural temporal coding in the current study is likely because of the low average stimulation rate of the FAST strategy.

The observation of an improvement in neural ITD sensitivity without an improvement in neural temporal coding in the IC, raises a question about the neural mechanism behind the limited temporal processing and ITD sensitivity in CI listening. The perceptual limit of ITD sensitivity at high pulse rates is similar in some respects to the limit of monaural rate discrimination in CI users. Most CI listeners can discriminate the rate of periodic pulse trains up to $\sim 300$ pps (Moore and Carlyon, 2010). Similarly, most CI users show a degradation in perceptual ITD sensitivity for pulse rates above $\sim 300$ pps (Kan and Litovsky, 2015; Laback et al., 2015). Ihlefeld et al. (2015) showed a small correlation between monaural rate discrimination and ITD discrimination performance across different pairs of electrodes in the same subjects, consistent with the view that temporal coding and ITD sensitivity are dependent on a common neural mechanism. In contrast, the current study found better neural ITD coding without a concomitant improvement in temporal coding in the IC of ED-CS rabbits. At the primary sites of binaural interaction in the MSO and lateral superior olive (LSO) temporal coding and ITD sensitivity are closely linked because precise synchrony of neural responses to the binaural stimuli is required for the operation of the coincidence detector neurons that create ITD sensitivity (Batra and Yin, 2004). However, this is not the case in the IC, where ITD is coded in the average firing rate, while temporal coding may be limited by the time constants of synaptic integration of the projections from MSO/LSO to the IC. In addition, the IC also receives inputs from the cochlear nuclei that are subject to temporal limitations of their own. Thus, the dissociation between improvements in ITD and temporal coding in the IC of ED-CS animals is consistent with the functional anatomy of the auditory brainstem.

Mechanisms for effect of CI stimulation during development The restoration of ITD sensitivity observed in IC neurons with chronic CI stimulation in early-deaf animals could be mediated by neural plasticity at any site along the ascending auditory pathway, just as the degradation in unstimulated animals could be caused by damage at any site. Although the reduction in SGN counts and demyelination of auditory nerve fibers can contribute to the degraded temporal coding associated with deafness (Hardie and Shepherd, 1999; Resnick et al., 2018), this effect is not limited to early-onset deafness (Shepherd and Javel, 1997). In addition, we did not find a preventative effect of chronic CI stimulation on the degeneration of SGNs such as that observed in ototoxically deafened cats in some studies (Leake et al., 1991, 1999). Our results are more consistent with the findings of Coco et al. (2007), who also failed to observe an improvement in SGN survival in ototoxically deafened and chronically stimulated cats. These differences might be explained by the state of the SGN population at the onset of chronic stimulation. A significant degeneration may have already occurred before the onset of chronic stimulation in studies that did not find a reversal in SGN degeneration. In any case, the restoration of neural ITD sensitivity in the IC in the current study cannot be explained by improved SGN survival in the stimulated group.

Structural abnormalities in the endbulb synapses between auditory nerve fibers and spherical bushy cells of the cochlear nucleus in congenitally-deaf cats can be reversed by chronic CI stimulation during development (Ryugo et al., 2005; O’Neil et al., 2010). Because these synapses are specialized for transmission of precise temporal information, abnormalities in their morphology might degrade temporal coding and ITD processing in the MSO. Tirko and Ryugo (2012) showed that the reduction in the size of excitatory synaptic boutons and in the number of inhibitory synapses onto MSO principal cells which occurs in the congenitally-deaf cats can be partly reversed by chronic bilateral CI stimulation during development. The dissociation between neural temporal and binaural coding found in the IC suggest that a moderate level of restoration in temporal and binaural coding might be occurring in the MSO.

\section{Implication for human performance and rehabilitation}

Bilateral CIs have become the standard of care for children with congenital deafness. Children who receive simultaneous bilateral CIs at an early age show a partial reversal in the degraded cortical representation of ITDs because of deafness (Easwar et al., 2017). Similarly, bilateral CI stimulation starting at an early age can help improve perception of binaural cues, although not nearly to the level of NH peers (Gordon et al., 2014). In contrast, children who experience unilateral hearing can develop a strong aural preference (Kral et al., 2013) that negatively affects ITD sensitivity (Tillein et al., 2016). Results from the current study provide further evidence for the importance of appropriate auditory experience during development in early deafness. Our "chronic" stimulation only lasted $25 \mathrm{~h} /$ week ( $5 \mathrm{~h} / \mathrm{d}, 5 \mathrm{~d} /$ week) yet, possibly in combination with behavioral training, achieved a significant therapeutic effect in restoring binaural sensitivity. Longer duration of stimulation with a more meaningful behavioral context might result in even better outcomes.

Children in the studies cited above were implanted with clinical devices and processors that are not designed to deliver ITD 
cues as the FAST processors used in the current experiment. Currently, clinical devices only provide envelope ITD cues. Whether further improvement in binaural sensitivity can be achieved with better access to ITD cues is unknown. Results from the current study alone do not show that precise ITD cues in the bilateral CI stimulation are necessary for reversing the degradation in ITD sensitivity in early-onset deafness. However, Fallon et al. (2015) found no significant restorative effect of bilateral CI stimulation on binaural sensitivity of IC neurons with CIS stimulation in anesthetized cats. On the other hand, our results and those of Vollmer et al. (1999) also suggest that temporal properties of the electrical stimuli might be important for the development of temporal coding in the central auditory pathway. A processing strategy that can deliver precise binaural cues without a reduction in stimulation rate might be necessary to restore both temporal and binaural sensitivity with CIs. Recent studies have shown that introduction of short inter-pulse intervals in high-rate pulse trains can enhance ITD sensitivity in both human perception (Srinivasan et al., 2018, 2020) and IC neurons (Buechel et al., 2018).

In summary, we showed partial, but significant, restoration of neural ITD sensitivity in the auditory midbrain of early-deaf animals that received chronic bilateral stimulation during development. The restoration in neural coding of electrical stimuli was specific to ITDs and no improvement in temporal coding was found.

\section{References}

Batra R, Yin TC (2004) Cross correlation by neurons of the medial superior olive: a reexamination. J Assoc Res Otolaryngol 5:238-252.

Baumgärtel RM, Hu H, Kollmeier B, Dietz M (2017) Extent of lateralization at large interaural time differences in simulated electric hearing and bilateral cochlear implant users. J Acoust Soc Am 141:2338.

Buechel BD, Hancock KE, Chung Y, Delgutte B (2018) Improved neural coding of ITD with bilateral cochlear implants by introducing short interpulse intervals. J Assoc Res Otolaryngol 19:681-702.

Chung Y, Hancock KE, Nam SI, Delgutte B (2014) Coding of electric pulse trains presented through cochlear implants in the auditory midbrain of awake rabbit: comparison with anesthetized preparations. J Neurosci 34:218-231.

Chung Y, Hancock KE, Delgutte B (2016) Neural coding of interaural time differences with bilateral cochlear implants in unanesthetized rabbits. J Neurosci 36:5520-5531.

Chung Y, Buechel BD, Sunwoo W, Wagner JD, Delgutte B (2019) Neural ITD sensitivity and temporal coding with cochlear implants in an animal model of early-onset deafness. J Assoc Res Otolaryngol 20:37-56.

Coco A, Epp SB, Fallon JB, Xu J, Millard RE, Shepherd RK (2007) Does cochlear implantation and electrical stimulation affect residual hair cells and spiral ganglion neurons? Hear Res 225:60-70.

Cohen J (1988) The analysis of variance and covariance. In: Statistical power analysis for the behavioral sciences, Ed 2, Chapter 8. Hillsdale: L. Erlbaum Associates.

Cornell Lab of Ornithology (2001) The diversity of animal sounds. Ithaca, New York:Macaulay Library.

Easwar V, Yamazaki H, Deighton M, Papsin B, Gordon K (2017) Cortical representation of interaural time difference is impaired by deafness in development: evidence from children with early long-term access to sound through bilateral cochlear implants provided simultaneously. J Neurosci $37: 2349-2361$.

Elliott L, Hershberger W (2007) The songs of insects. Boston: Houghton Mifflin Harcourt.

Fallon J, Irving S, Wise A, Irvine DR (2015) Effects of cochlear implant use on binaural processing. Assoc Res Otolaryngol Abstr 38-726.

Foote RH, Carney EW (2000) The rabbit as a model for reproductive and developmental toxicity studies. Reprod Toxicol 14:477-493.

Foss I, Flottorp G (1974) A comparative study of the development of hearing and vision in various species commonly used in experiments. Acta Otolaryngol 77:202-214.
Gordon KA, Deighton MR, Abbasalipour P, Papsin BC (2014) Perception of binaural cues develops in children who are deaf through bilateral cochlear implantation. PLoS One 9:e114841.

Hancock KE, Noel V, Ryugo DK, Delgutte B (2010) Neural coding of interaural time differences with bilateral cochlear implants: effects of congenital deafness. J Neurosci 30:14068-14079.

Hancock KE, Chung Y, Delgutte B (2012) Neural ITD coding with bilateral cochlear implants: effect of binaurally coherent jitter. J Neurophysiol 108:714-728.

Hardie NA, Shepherd RK (1999) Sensorineural hearing loss during development: morphological and physiological response of the cochlea and auditory brainstem. Hear Res 128:147-165.

Heffer LF, Fallon JB (2008) A novel stimulus artifact removal technique for high-rate electrical stimulation. J Neurosci Methods 170:277-284.

Hossain S, Goldsworthy RL (2018) Factors affecting speech reception in background noise with a vocoder implementation of the FAST algorithm. J Assoc Res Otolaryngol 19:467-478.

Ihlefeld A, Carlyon RP, Kan A, Churchill TH, Litovsky RY (2015) Limitations on monaural and binaural temporal processing in bilateral cochlear implant listeners. J Assoc Res Otolaryngol 16:641-652.

Isaiah A, Vongpaisal T, King AJ, Hartley DE (2014) Multisensory training improves auditory spatial processing following bilateral cochlear implantation. J Neurosci 34:11119-11130.

Ishiyama G, Geiger C, Lopez IA, Ishiyama A (2011) Spiral and vestibular ganglion estimates in archival temporal bones obtained by design based stereology and Abercrombie methods. J Neurosci Methods 196:76-80.

Kan A, Litovsky RY (2015) Binaural hearing with electrical stimulation. Hear Res 322:127-137.

Kral A, Heid S, Hubka P, Tillein J (2013) Unilateral hearing during development: hemispheric specificity in plastic reorganizations. Front Syst Neurosci 7:93.

Laback B, Egger K, Majdak P (2015) Perception and coding of interaural time differences with bilateral cochlear implants. Hear Res 322:138-150.

Leake PA, Hradek GT, Rebscher SJ, Snyder RL (1991) Chronic intracochlear electrical stimulation induces selective survival of spiral ganglion neurons in neonatally deafened cats. Hear Res 54:251-271.

Leake PA, Hradek GT, Snyder RL (1999) Chronic electrical stimulation by a cochlear implant promotes survival of spiral ganglion neurons after neonatal deafness. J Comp Neurol 412:543-562.

Litovsky RY, Parkinson A, Arcaroli J (2009) Spatial hearing and speech intelligibility in bilateral cochlear implant users. Ear Hear 30:419-431.

Litovsky RY, Jones GL, Agrawal S, van Hoesel R (2010) Effect of age at onset of deafness on binaural sensitivity in electric hearing in humans. J Acoust Soc Am 127:400-414.

Moore BCJ, Carlyon RP (2010) Perception of pitch by people with cochlear hearing loss and by cochlear implant users. In: Pitch: neural coding and perception (Plack CJ, Oxenham AJ, Fay RR, eds), pp 234-277. New York: Springer.

Murphy J, O’Donoghue G (2007) Bilateral cochlear implantation: an evidence-based medicine evaluation. Laryngoscope 117:1412-1418.

O’Neil JN, Limb CJ, Baker CA, Ryugo DK (2010) Bilateral effects of unilateral cochlear implantation in congenitally deaf cats. J Comp Neurol 518:2382-2404.

Ramprashad F, Landolt JP, Money KE, Laufer J (1984) Morphometric study of the cochlea of the rabbit (Oryctulagus cuniculus). Can J Zool 62:24762481.

Resnick JM, O’Brien GE, Rubinstein JT (2018) Simulated auditory nerve axon demyelination alters sensitivity and response timing to extracellular stimulation. Hear Res 361:121-137.

Ryugo DK, Kretzmer EA, Niparko JK (2005) Restoration of auditory nerve synapses in cats by cochlear implants. Science 310:1490-1492.

Salloum CA, Valero J, Wong DD, Papsin BC, van Hoesel R, Gordon KA (2010) Lateralization of interimplant timing and level differences in children who use bilateral cochlear implants. Ear Hear 31:441-456.

Shepherd RK, Javel E (1997) Electrical stimulation of the auditory nerve. I. Correlation of physiological responses with cochlear status. Hear Res 108:112-144.

Smith ZM (2010) Improved sensitivity to interaural time differences with the FAST coding strategy. 11th International Conference on Cochlear Implants and Other Implantable Auditory Technologies. Stockholm: Sweden. 
Smith ZM, Delgutte B (2007) Sensitivity to interaural time differences in the inferior colliculus with bilateral cochlear implants. J Neurosci 27:67406750 .

Smith ZM, Kan A, Jones HG, Buhr-Lawler M, Godar SP, Litovsky RY (2014) Hearing better with interaural time differences and bilateral cochlear implants. J Acoust Soc Am 135:2190-2191.

Snyder R, Leake P, Rebscher S, Beitel R (1995) Temporal resolution of neurons in cat inferior colliculus to intracochlear electrical stimulation: effects of neonatal deafening and chronic stimulation. J Neurophysiol 73:449-467.

Srinivasan S, Laback B, Majdak P, Delgutte B (2018) Introducing short interpulse intervals in high-rate pulse trains enhances binaural timing sensitivity in electric hearing. J Assoc Res Otolaryngol 19:301-315.

Srinivasan S, Laback B, Majdak P, Arnoldner C (2020) Improving interaural time difference sensitivity using short inter-pulse intervals with amplitude-modulated pulse trains in bilateral cochlear implants. J Assoc Res Otolaryngol 21:105-120.

Tillein J, Hubka P, Syed E, Hartmann R, Engel AK, Kral A (2010) Cortical representation of interaural time difference in congenital deafness. Cereb Cortex 20:492-506.
Tillein J, Hubka P, Kral A (2016) Monaural congenital deafness affects aural dominance and degrades binaural processing. Cereb Cortex 26:1762-1777.

Tirko NN, Ryugo DK (2012) Synaptic plasticity in the medial superior olive of hearing, deaf, and cochlear-implanted cats. J Comp Neurol 20:2202-2217.

van Hoesel RJ, Tyler RS (2003) Speech perception, localization, and lateralization with bilateral cochlear implants. J Acoust Soc Am 113:1617-1630.

van Hoesel RJ, Jones GL, Litovsky RY (2009) Interaural time-delay sensitivity in bilateral cochlear implant users: effects of pulse rate, modulation rate, and place of stimulation. J Assoc Res Otolaryngol 10:557-567.

van Loon MC, Ramekers D, Agterberg MJ, de Groot JC, Grolman W, Klis SF, Versnel H (2013) Spiral ganglion cell morphology in guinea pigs after deafening and neurotrophic treatment. Hear Res 298:17-26.

Vollmer M, Snyder RL, Leake PA, Beitel RE, Moore CM, Rebscher SJ (1999) Temporal properties of chronic cochlear electrical stimulation determine temporal resolution of neurons in cat inferior colliculus. J Neurophysiol 82:2883-2902.

Vollmer M, Leake PA, Beitel RE, Rebscher SJ, Snyder RL (2005) Degradation of temporal resolution in the auditory midbrain after prolonged deafness is reversed by electrical stimulation of the cochlea. J Neurophysiol 93:3339-3355. 